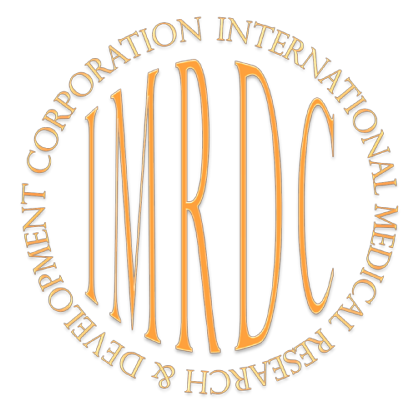

\title{
Creativity as a Determinant of the Development of Homo Sapiens
}

\author{
Alexander G. Kruglov, PhD, ScD \\ Central Research Institute of Radiation Diagnosis \\ Moscow, the Russian Federation
}

\begin{abstract}
This work was undertaken to determine the genesis and role of creativity $(\mathrm{Cr})$ in the formation of mental qualities that gave Homo sapiens (HS) the evolutionary advantages in intra- and interspecific competition during the period of the intraspecific bifurcation of hominids on the border of the Middle and Upper Paleolithic. Creativity allowed HS to design the adaptive forms of purposeful behavior corresponding to the conditions and the degree of uncertainty, and create stable mental constructs, in the absence of perceptual sources, that do not require reactive behavior. Visualization of a target image, which originally had an applied and instructional value in the process of semantic filling, was transformed into a symbol, which, getting the qualities of the perceptual source, loses its connection to the primary value and initiates the creation of qualitatively new needs for hominids: bilateral (direct and inverse) relations in the system "subject-symbol." The ability to produce the prognostic hypothesis with expansion of the operating range of the HS mind allowed the ability to search and change the tactics of an adaptive behavior, which gave the results: improvements in quality of life and an increase in life expectancy (genetically fixed); domination in intraand interspecific competition; the emergence of new operating systems of the psyche, including the emergence and development of symbolic thought. As a criterion of creativity, with the potential ability for quantitative measurement, we propose the value of deviation of creative oscillations ("proposal" of a creative individual) from the boundaries between stereotypes: for the cognition sphere, a deviation from the border between recognizable and unrecognizable; for the social sphere, between acceptable and unacceptable. (Int J Biomed. 2016; 6(4):298-302.)
\end{abstract}

Key Words: creativity • genetic mutation • shizotimiya $\bullet$ interspecific competition

The aim of our study was to determine the genesis and the role of creativity of HS in the formation of adaptive forms of behavior in conditions of intra- and interspecific competition.

To build a model of creativity as a mental function, along with the works of cited authors, we used our previously published materials. ${ }^{[1]}$ Creativity (a species-specific feature of the psyche of HS compensating the lack of biological adaptability), as the ability to produce the predictive hypotheses that cannot be derived directly from the initial conditions, formed about 50,000 years ago, presumably as a result of genetic mutation ${ }^{[2]}$ that does not exclude the possibly interfering evolutionary and other exogenous factors. It is necessary to emphasize the existence of various exogenous (including biogenic) factors which change the external behavioral manifestations of mental activity not only in humans, but also in other

Corresponding author: Alexander G. Kruglov, PhD, ScD Central Research Institute of Radiation Diagnosis. Moscow, the Russian Federation. E-mail: krag48@mail.ru species. For example, Toxoplasma gondii alters the behavior of rodents up to eliminating the instinct of self-preservation while maintaining newly acquired psychic qualities after full recovery ${ }^{[3]}$ In the available literature, we have not found works about the genetic fixation of the new mental properties in rodents that underwent toxoplasmosis. It was noted that there is a similarity in some syndromic forms of schizophrenia and toxoplasmosis in humans, ${ }^{[4]}$ as well as a significant correlation between toxoplasmosis and expressed anxiety. ${ }^{[5]}$ Not having sufficient grounds to assert the identity of states defined as schizophrenia with the effects of toxoplasmosis, we would like to emphasize their considerable syndromic similarity. It is possible that Toxoplasma gondii, in the considered historical period, could not be the single biogenic factor with potential effects on the brain (mind) of man.

We are interested in an historical period having significant time assumptions (for thousands, sometimes tens of thousands of years) as a result of new archaeological finds - the completion of the Middle Paleolithic (300,00040,000 years ago), the time when the tribal system replaced 
the primitive herd. This is a period of significant rapid climate changes during the ice age ${ }^{[6]}$ increased intra- and interspecific competition, disappearance of species that did not have a sufficient range of adaptability, and the emergence of the modern physical type - Cro-Magnon man (HS).

HS existed in small groups with a dominant leader, the hierarchical distribution of functions and benefits, and the suppression of individual traits. The emergence of communities, the development of intergeneric relationships, and exogamous marriage in the face of increasing competition for food resources with competing subspecies, Homo Neanderthalensis $(\mathrm{HN})$, led to a blurring of the boundaries between the possible and permissible, an increase in the degree of uncertainty on the boundaries of hierarchic subsystems (genuses) within and outside of the community. We understand uncertainty as the variability of choice for a variety of alternatives and the absence of clear criteria of optimality and efficiency. There are conceptions of uncertainty as a fundamental property of nature. ${ }^{[7]}$

Benefits that include the ability to search and change tactics while ensuring competitiveness within and between species, and an increase in the probability of the occurrence of posterity (inheritance and accumulation of parent qualities) reconcile the tolerant individuals to uncertainty.

Emergence of new mental characteristics $(\mathrm{Cr})$ allows us to construct the target images, adaptive forms of purposeful behavior appropriate for the condition and the degree of uncertainty. The abstracted images of hunting objects with detailed scenes achieving the desired results (the cave of Altamira $)^{[8]}$ demonstrate the HS ability to create consistent themes of purposeful behavior, achieving future results, and the image of the goal (IG), which has no perceptual sensory source in the process of its creation. Through the operating systems of the psyche, presumably integrable by $\mathrm{Cr}$, the structure of purposeful behavior is created, which includes IGs that are outside the boundaries of the immediate satisfaction of vital needs. In other words, a new feature of the species $(\mathrm{Cr})$ allows us to create stable mental constructs in the absence of a perceptual source and does not require a reactive adaptive behavior. In particular, an abstracted and coded IG is created, which actualizes the need to achieve this goal by following purposeful behavior that ensures satisfaction of this need, in the absence of which the "anxious waiting" state is formed. Adaptive behavior gets a vector of satisfaction for a qualitatively new need: the relationships with the coded and visualized IG, which is not associated with reactive behavior. We believe that the visualization of IG, having initially utilitarian and instructional value, inevitably becomes the subject of supplements, discussion, verbalization, and interpretations (ie, semantic content), transforming into a symbol (image + sense) ${ }^{[9]}$ includes:

Cr, implying the capacity for symbolic thinking,

- a selective attention to contradictions;

- deviations from the standard;

- a tendency to allocate the minor, non-obvious features of the information field as reference by following construction of an integrated mental product (image); and
- the ability to design a set of solutions on the basis of monosemantic data (divergent thinking). ${ }^{[10]}$

Creative individuals are characterized by the identification of the subjective "I" with an imaginary (external) objective "I", by abstraction, and the "transfer" (imagination, empathy) of personal properties to a third party (an external object), a kind of schism of integral personal presentation. ${ }^{[1]}$

We believe that the appearance of $\mathrm{Cr}$ created an evolutionary bifurcation that gave a branch of HS an evolutionary advantage that enabled it to produce the divergent forecasting models and generate the adaptive behaviors adequate to different degrees of uncertainty. A possible effect of cumulative accumulation of qualitative changes of the psyche is the appearance at the end of the Middle Paleolithic (about 40,000 years ago) of the samples of fine art of CroMagnons around the habitat, signs of the cultural environment, and domestication and involvement of dogs in economic activity (about 33,000 years ago). ${ }^{[12]}$

In HS, in incomplete or "open" vitally important situations to the integration of new elements (deficiency), there is an inevitable development of the state of dissatisfaction, a deficit of that condition which is required to maintain a homeostatic balance; and the need to fill the missing fragments in the imaginative mapping of reality is formed. We believe that $\mathrm{Cr}$ provides a constructive way to complete the deficient image of reality by using the brain's operating systems (thinking, imagination, memory, etc.). Thus, anticipatory and perspective designing of an integral image of a future result (IG), completion of which is experienced as "insight," is realized, as well as the method of meeting the arising need, which reduces the level of risk in the process of achieving the result with elimination of "anxious waiting," which leads to a relationship of equilibrium with the object (IG).

Individuals (tolerant to uncertainty) design (through Cr) the abstracted IGs which are visualized through graphic forms with acquisition of more sophisticated color, contour, perspective, and plot, and transformed into art forms - the symbols. The result is unique to the species: the ability to create stable mental constructs with the following projection into external environments as symbolic forms, visualization of which as artistic images appeals to the arsenal of ideas. These forms are perceived in the inverse projection (reflection of reflection) as objectively existing (beliefs, myths, etc.). An image having the applied instructional value becomes an independent mental construct (symbol), acquiring the quality of the perceptual source, which creates an afferent information flow and motivates the formation of the mental activity vector to achieve the goal: the parametric balance with the symbol. The symbol dissociation, eliminating the "image" (category of representativity), retains only "sense" as the addressee of subject appeal (ie, symbol, imagery) becomes exclusively virtual, without losing the qualities of objectively existing for the subject. Obviously, this is the mechanism that forms the coded images of the primary myths initiated by the abstractive (symbolic) IG, followed by transformation (complications, interference, interpretation by the second signaling system) in totems, cults, ideologies, etc. In certain conditions (resonance, including induction), this construction, being entirely a 
product of the second signaling system, can acquire the properties of the dominant focus with appropriate mechanisms (neural, metabolic, and behavioral) of implementation. The combination of these constructs, creating a system of representations (virtual system of ethical coordinates) about relationships within and outside society, reduces the level of uncertainty, creates and regulates the coordinate system of the social environment and its relations with the outside, and reduces the level of suspense. Intra-group relations of the ethical coordinate construct reduce the degree of uncertainty (the creator of social negentropy), namely, relations between a creative person resistant to uncertainty (conditional priest, ideologist - "divergent") and another person, the advantage of which is the ability to adopt and implement adequate solutions in the current coordinate system (conditional leader"convergent"), as well as interference of functions in one individual. These will be issues for the next study.

According to modern ideas, the meeting of 2 subspecies of Homo: HN (natives) and HS (aliens from the south) occurred in Eurasia about 50,000 years ago. HN and HS are the most adapted modern gregarious predators. $\mathrm{HN}$ and $\mathrm{HS}$ lived together on the "feeding" landscapes of Eurasia for 6,000 years in conditions of increasing competition (with an increase in population) for food resources. HS, having a new feature of the species $(\mathrm{Cr})$, compared with $\mathrm{HN}$, acquired a much greater range of adaptive behaviors, as well as a strategic advantage in conditions of interspecific competition, which resulted in dominating and then crowding out the competitive subspecies (HN). It should be noted that this particular historical period (date of occurrence from 50,000 to 138,000 years ago) ${ }^{[13]}$ is a time when the Y-chromosomal Adam occurred, whose actively functioning genes (without alleles in the X-chromosome) are presented only in the genotype of heterogeneous gender, appearing phenotypically only in representatives of heterogametic sex.

The vector of subsequent analysis is based on nearzero probability of female authorship as the creator of the cave art images that had practical importance and required the knowledge, skills and experience of direct contact with the image object that was not familiar to the average woman, regardless of historical era. Regardless of the prevalence (in different historical periods) of matriarchal or patriarchal society, while hunting the male groups were structured organizations with the undisputed leader (possibly only during the hunting time) and the distribution of functions on the pack model. Anthropometrically, psychologically and physiologically, the appropriate psychosomatic conditions are more typical for men.

We believe that a point mutation in a Y-linked gene led to development of a new quality of the psyche - creativity. Sexual differences for mammals, including humans, are provided by a pair of sex chromosomes (XX and XY). Reduction or translocation of genes, useless or harmful to the females, led to the separation and concentration of genes, providing the development of physiology and psychology of the male type. The SRY gene located on the short arm of the Y-chromosome plays the most important role in initiating male sex determination. The peculiarity of the human Y-chromosome is a short length of regions, which are exchanged with loci of other chromosomes, in particular, with the X-chromosome, except for the pseudoautosomal regions occupying about $5 \%$ of the Y-chromosome's length.

Sequencing the genome of the Neanderthal in comparison with genome of the Cro-Magnon man, in the framework of modern features, revealed a difference between them of $0.16 \%$. Not found in Neanderthals were genes associated with mental activity and energy exchange, having adaptive character, ${ }^{[14]}$ and genes associated with the development of schizophrenia and bipolar disorder, ${ }^{[15]}$ as well as a reduced number of genes on the site responsible for the development of speech. ${ }^{[16]}$ Genes that have been found in CroMagnons and are absent in Neanderthals and chimpanzees emerged during evolutionary bifurcation (or became the cause of it). The number of nucleotide substitutions that have semantic meaning, is $1 \%$ of the total differences between the human and chimpanzee genome (ie, 1 million base pairs, 78 single-nucleotide differences) with the concentration of these differences in the DNA regions encoding 5 genes. ${ }^{[17]}$ We believe that the focused search in this area can reveal a substrate (genetic) basis of $\mathrm{Cr}$.

Genetic variations associated with increased risk of schizophrenia and bipolar disorder were found in a large sample of HS. ${ }^{[18]}$ In a creative environment, variations in genes associated with an increased likelihood of these diseases are higher by $17 \%-25 \%$.

A key feature of schizoid thinking, ${ }^{[19]}$ along with a tendency toward complex mental operations (generalization, abstraction) is choosing as reference points the insignificant, latent signs, and then using them for generalization and abstraction compared to the most frequent stereotypes entrenched in the experience. In particular, there is a change in the logical course of judgment, when the conventional story and the logic of the sequence are understood, but not accepted, and the formation of the judgment is formed in several directions. All of this leads to the creation of standalone logic with non-obvious conclusions that differ from the findings of individuals having the same original data. In this paper we do not discuss changes in thinking that reached the level of the marked clinical manifestations. We are interested in basic mechanisms of changes in thinking that give the opportunity to find qualitatively new forms of adaptive behavior, avoiding stereotypes. We would like to emphasize the common features for the schizoid and creative personalities: an inability to filter out irrelevant information; a propensity to build mental (associative, shaped) structures that differ from the social standards; cognitive disinhibition; sthenic emotions; splitting of the personal views, as well as other features confirming the similarity of cognitive processes involved in creativity and mental illness. Through MRI, ${ }^{[20]}$ it was found that creative individuals and groups with the confirmed signs of schizothymia, when solving cognitive tasks, displayed the same objective equivalents: deactivation in the right parietal region and precuneus - in the areas responsible for concentration.

A study of single nucleotide polymorphisms of genes of modern man found 8 genetically determined variants of the 
clinical forms of schizophrenia, which depend on the structure of clusters. In addition, if each gene had a tenuous connection with the development of the disease, a cluster association increased the risk by $70 \%-100 \% \cdot{ }^{[21]}$ Regarding the combination of genes that have a limited number of variations, it was stated that there is the simultaneous presence of a predisposition to the schizoid state with characteristic changes of thinking and of creativity. It should be noted that the average life expectancy (26-32.3 years) ${ }^{[6]}$ in this historical period at lowprogredient forms of endogenous diseases (debuting in 20-25 years) reduced the probability, because of high-risk habitats, of reaching the final or even psychotic deployed stages. Thus, new features of the psyche for hominids, acquired as a result of a genetic mutation or other reasons, with schizoid initiation in the mature age (for the historical period) can form deviations (in the modern sense, disorders) in thinking, such as ideas of influence, relationships, harassment, and mania states in varying degrees of severity. In other words, schizoid accentuation or endogenous disease in the prodromal stage may lead to the construction of sustainable ideas about an exogenous impact (in relation to the individual), the presence of an external object, perceived by the individual perceptually with the corresponding effects on the subject. This stimulates the creation, development, and interference of logic circuits forming and unifying the perceived metamorphosis. The result is the construction of predictive models of development and outcome of the situation, with variations of personal and social behavior. We believe it possible, among other things, to consider the development of a second signaling system as a need to express and describe something that has no obvious perceptual source for the audience. Thus, a stable endogenous psychic structure, becoming a representation, is transformed by a subject into visualized and verbal forms (as the semantic content - symbols), transformed into the belief (an inverse projection) and initiates the adaptive forms of behavior in the relationship system: an individual-a symbol (in certain circumstances: society-symbol). Artistic images, originally of an applied nature, with the accumulation and complexity of semantic content, are dissociated from an initial sense and acquire an independent significance, becoming abstract symbols forming a qualitatively new demand (and forms of adaptive behavior): parametric and homeostatic equilibrium of relationship with the symbol.

The logic of the discussion leads to the conclusion that just the carriers of $\mathrm{Cr}$, having the above properties of the psyche (sthenic emotions, the tendency to oscillation) with deviation from the allowable and acceptable borders, allow us to find new optima of the individual and system functioning (ie, to determine the vectors of adaptation for society to the changing parameters of an external [or internal] environment). As a criterion of creativity, with the potential ability for quantitative measurement, we propose the value of deviation of creative oscillations ("proposal" of a creative individual) from the boundaries between stereotypes: for the cognition sphere, a deviation from the border between recognizable and unrecognizable; for the social sphere, between acceptable and unacceptable.

The described time interval, ending the Middle
Paleolithic and beginning the Upper Paleolithic, for HS is a time of formation (as a result of improved quality of life) of a more improved structure of biological age: late ripening late aging (for $\mathrm{HN}$ : early ripening - premature aging) with an increase in life expectancy and fixing the potential ability for adaptive variation at the genetic level, which increased the average life expectancy in the Upper Paleolithic (the next stage after a long break is the Middle Bronze Age). ${ }^{[6]}$ We believe that an increase in life expectancy to more than 30 years for the $\mathrm{Cr}$ carriers in the prodrome of endogenous disease (unlike accentuation) could reveal a side effect of one of the variants of the genetic mechanism for an increase in population adaptability: an emergence of the expanded forms of endogenous diseases, which dramatically increase risks for their carriers.

\section{Conclusion}

Creativity that expanded the adaptive capacity of HS - as the ability to assimilate and process the volume of information in excess of the standards and to allocate the latent signs of environment as reference, as well as a cognitive disinhibition that increased the associative range and split of the personal representations with formation of judgments in accordance with the convergent type-has formed a unique ability to create sustainable mental constructs nonassociated with reactive behavior, combining image and meaning (projected into the external environment by the individual) in the form of artistic images. The subsequent transformation into independent symbolic forms, perceived as the external objects, forms a need in bilateral relations, initiating an adaptive behavior that balances the relationships in the "subject-a symbol" system. The results of the described process are advantages and then domination in intra- and interspecific competition in conditions of uncertainty, as well as improvements in the quality and expectancy of life for HS. As a criterion of creativity, with the potential ability for quantitative measurement, we propose the value of deviation of creative oscillations ("proposal" of a creative individual) from the boundaries between stereotypes: for the cognition sphere, a deviation from the border between recognizable and unrecognizable; for the social sphere, between acceptable and unacceptable.

\section{References}

1. Kruglov AG, Vasilyev A, Sherman V. Human dynamic homeostasis control matrix in the norm with psychophysiological aspects. New York: IMRDC; 2016, 56 pp. 2. Klein RG. The human career. Human biological and cultural origins. Chicago: The University of Chicago Press; 1989, 524 pp. 3. Ingram WM, Goodrich LM, Robey EA, Eisen MB. Mice infected with low-virulence strains of Toxoplasma gondii lose their innate aversion to cat urine, even after extensive parasite clearance. PLoS One. 2013;8(9):e75246.

4. Torrey EF, Yolken RH. Toxoplasma gondii and schisophrenia . Emerg Infect Dis. 2003;9(11):1375-80.

5. Markovitz AA, Simanek AM, Yolken RH, Galea S, Koenen $\mathrm{KC}$, Chen $\mathrm{S}$, et al. Toxoplasma gondii and anxiety disorders in a community-based sample. Brain Behav Immun. 2015;43:192-7. 
6. Buzhilova AP. Homo sapiens - the history of the disease. Moscow: the Russian Academy of Sciences, Institute of Archaeology; 2005, 320 pp. [in Russian].

7. Kravchenko AO. Abstracts on uncertainty. UNIVERSITATES. (Nauka I Prosveshchenie), 2014;4:14 -19.

8. Devlet E. Altamira: at the origins of art. M.: 2004, 142 pp.

9. Losev AF. Essays on ancient symbolism and mythology. M.: Misl; 1993, 962 pp. [in Russian].

10. Guilford JP. Way Beyond the IQ. Buffalo (NY): Creative Education Foundation;1977, 192 pp.

11. Philosophy: Encyclopedic Dictionary. Ed. AA Ivin. M .: Gardariki; 2004, 1072 pp. [in Russian].

12. Ovodov ND, Crockford SJ, Kuzmin YV, Higham TF, Hodgins GW, van der Plicht J. A 33,000-year-old incipient dog from the Altai Mountains of Siberia: evidence of the earliest domestication disrupted by the Last Glacial Maximum. PLoS One. 2011;6(7):e22821.

13. Poznik GD, Henn BM, Yee MC, Sliwerska E, Euskirchen GM, Lin AA, et al. Sequencing Y chromosomes resolves discrepancy in time to common ancestor of males versus females. Science. 2013;341(6145):562-5.

14. Burbano HA, Hodges E, Green RE, Briggs AW, Krause $\mathrm{J}$, Meyer M, et al. Targeted investigation of the Neandertal genome by array-based sequence capture. Science. 2010 May 7;328(5979):723-5.
15. Srinivasan S, Bettella F, Mattingsdal M, Wang Y, Witoelar A, Schork AJ, et al. Genetic Markers of Human Evolution Are Enriched in Schizophrenia. Biol Psychiatry. 2016; 80(4):284-92. 16. Vernot B, Akey JM Resurrecting surviving Neanderthal lineages from modern human genomes. Science. 2014; 343(6174):1017-21.

17. Green RE, Krause J, Briggs AW, Maricic T, Stenzel U, Kircher M, et al. A draft sequence of the Neandertal genome. Science. 2010;328(5979):710-22.

18. Power RA, Steinberg S, Bjornsdottir G, Rietveld CA, Abdellaoui A, Nivard MM, et al. Polygenic risk scores for schizophrenia and bipolar disorder predict creativity. Nat Neurosci. 2015;18(7):953-5.

19. Zeigarnik BV. Pathopsychology. M.MGU; 1986,287 pp.[in Russian].

20. Fink A, Grabner RH, Benedek M, Reishofer G, Hauswirth $\mathrm{V}$, Fally $\mathrm{M}$, et al.The creative brain: investigation of brain activity during creative problem solving by means of EEG and FMRI. Hum Brain Mapp. 2009;30(3):734-48.

21. Arnedo J, Svrakic DM, Del Val C, Romero-Zaliz R, Hernández-Cuervo H; Molecular Genetics of Schizophrenia Consortium, Fanous AH, Pato MT, Pato CN, et al. Uncovering the hidden risk architecture of the schizophrenias: confirmation in three independent genome-wide association studies. Am J Psychiatry. 2015;172(2):139-53. 